\title{
Resonant Optical Antennas with
} Atomic-Sized Tips and Tunable Gaps Achieved by Mechanical Actuation and Electrical Control

\section{Journal Article}

Author(s):

Gruber, Cynthia M.; Herrmann, Lars O.; Bellido, Edson P.; Dössegger, Janine (D); Olziersky, Antonis; Drechsler, Ute; PueblaHellmann, Gabriel F.; Botton, Gianluigi A.; Novotny, Lukas (D); Lörtscher, Emanuel

\section{Publication date:}

2020-06-10

\section{Permanent link:}

https://doi.org/10.3929/ethz-b-000421266

\section{Rights / license:}

In Copyright - Non-Commercial Use Permitted

Originally published in:

Nano Letters 20(6), https://doi.org/10.1021/acs.nanolett.0c01072

\section{Funding acknowledgement:}

152944 - Field-enhanced chemical-optical spectroscopy platform for molecular sensing (SNF) 


\title{
Resonant Optical Antennas with Atomic-sized Tips and Tunable Gaps Achieved by Mechanical Actuation and Electrical Control
}

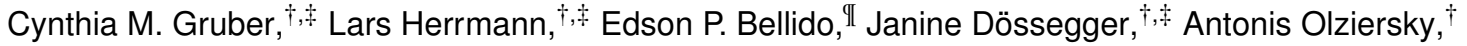 \\ Ute Drechsler, ${ }^{\dagger}$ Gabriel Puebla-Hellmann, ${ }^{\dagger}, \S$ Gianluigi A. Botton, ${ }^{\mathbb{I}}$ Lukas Novotny, ${ }^{\ddagger}$ and Emanuel \\ Lörtscher,,$\dagger$ \\ IBM Research - Zurich, Säumerstrasse 4, CH-8803 Rüschlikon, Switzerland, ETH Zürich, Photonics Laboratory \\ Hönggerbergring 64, CH-8093 Zürich, Switzerland, McMaster University, 1280 Main Street West, Hamilton, ON \\ L8S4M1, Canada, and University of Basel, St. Johanns-Ring 19, CH-4056 Basel, Switzerland \\ Received May 1, 2020; E-mail: eml@zurich.ibm.com
}

\begin{abstract}
Enhanced electromagnetic fields in nanometer gaps of plasmonic structures increase the optical interaction with matter, including Raman scattering and optical absorption. Quantum electron tunneling across sub-1-nm gaps, however, lowers these effects again. Understanding these phenomena requires controlled variation of gap sizes. Mechanically actuated plasmonic antennas enable repeatable tuning of gap sizes from the weak-coupling over the quantum-electron-tunneling to the direct-electrical-contact regime. Gap sizes are controlled electrically via leads that only weakly disturb plasmonic modes. Conductance signals show a near-continuous transition from electron tunneling to metallic contact. As the antenna's absorption cross-section is reduced, thermal expansion effects are negligible, in contrast to conventional breakjunctions. Optical scattering spectra reveal first continuous red shifts for decreasing gap sizes and then blue shifts below gaps of $0.3 \mathrm{~nm}$. The approach provides pathways to study opto- and electro-molecular processes at the limit of plasmonic sensing.
\end{abstract}

KEYWORDS: break junctions, surface plasmon polaritons, electron tunneling, electron energy-loss spectroscopy

Light-driven charge-density oscillations in metal nanostructures, whose quantized states are known as surface plasmon polaritons (SPPs), enable a strong enhancement of light-matter interactions in sub-diffraction volumes. In particular, the electro-magnetic field enhancements in the hot-spot region of plasmonic antennas allow for surface-enhanced spectroscopy and analyte sensing with a high sensitivity. ${ }^{1-7}$ A prerequisite for high field enhancements in antennas is a strong optical near-field coupling. This is achieved by nanometer-sized gaps combined with resonant excitation of the plasmonic modes through incident light. At sub-1-nm gap sizes, however, light-driven quantum tunneling allows surface charges to leak across the gap, thereby weakening the local electric fields. ${ }^{8,9}$ In addition, for many optical processes, "quenching" of incident, emitted or scattered photons occurs in close proximity to metallic surfaces through the conversion of photons into incoherent, nonradiative electron-hole excitations in the metal. A range of effects is thought to occur under high-field and high-field-gradient conditions, such as parametric amplification and cooling of molecular vibrations, ${ }^{10-12}$ most of which are still poorly understood. Hence, there is a general need for an optical testbed where such effects can be systematically studied through deterministic antenna design and continuous variation of gap sizes.

Reproducibly fabricating antennas with nanometer-sized gaps

\footnotetext{
${ }^{\dagger}$ IBM Research - Zurich

‡ETH Zürich

"McMaster University

${ }^{\S}$ University of Basel
}

is challenging, in particular when using top-down methods. ${ }^{8}$ Nevertheless, advances in electron-beam lithography (EBL), ${ }^{13}$ nanosphere lithography, ${ }^{14}$ nanoimprint lithography, ${ }^{15}$ or focusedion-beam milling ${ }^{16}$ allow tight control of feature sizes, including gap dimensions, in some cases down to a few nm. In contrast to bottom-up approaches, where the spacing between nanoparticles is often realized by tuning the length and binding properties of the molecular surface coatings ${ }^{1}$ or by electromigration of prepatterned leads, ${ }^{17,18}$ top-down methods provide greater flexibility for the antenna design. This enables resonant SPP-excitation conditions, or the integration of optical building blocks into more complex devices. In general, tuning of the gap size is impossible for antennas fabricated on a rigid substrate. Mechanically tunable gaps, however, can be achieved by utilizing scanning-probe techniques to vary the distance between surface and probe. In particular, scanning near-field optical microscopy in conjunction with electrical read-out has been used to investigate optical rectification, shot-noise, electron-plasmon interactions ${ }^{19-21}$ as well as plasmonic effects in strongly coupled resonant antennas ${ }^{22}$ down to the quantum tunneling regime. ${ }^{9,23}$ The main issues with most probe-based approaches, however, are the strong disturbances of plasmonic modes induced through the probe attachment, complex excitation geometries, and limited gap stabilities in the absence of feedback control. Mechanically controllable break-junctions (MCBJ) offer improved mechanical stability with a gap tunability from a few nanometers of separation down to full metallic contact, ${ }^{24,25}$ with less complex excitation geometries and less plasmonic disturbances, however, mostly at the cost of slow operation and reduced dynamic range. The underlying operation procedure is based on the controlled bending of a flexible substrate in a three-point bending mechanism (Fig. 1A) that elongates and finally breaks a constriction in a metal wire. In a forming and conditioning process, atomic-sized tips can be shaped thanks to the ductile response of metals to deformation. ${ }^{26}$ The stability of MCBJs is a consequence of the compact support of the structures, their nearby attachment to the common substrate and the high mechanical reduction factor (see below). While MCBJs are widely used for electronic transport measurements, ${ }^{27}$ they have only rarely been employed for optical experiments to date, e.g. for surface-enhanced Raman spectroscopy (SERS) ${ }^{28-30}$ at nanoscale dimensions for the visible regime. ${ }^{31}$ Here we combine the gap tunability of MCBJs with the top-down EBL patterning capability to realize resonant optical antennas whose gaps can be varied continuously by mechanical actuation. The detrimental thermal effects observed in conventional MCBJs are mitigated by replacing the bulky leads by much narrower and thinner wires, reducing the optical absorption cross-section accordingly. The antenna geometry is optimized for resonant coupling between the far-field and the tightly confined hot-spot. 
In MCBJs, the relationship between the gap separation, $\Delta d$, and the central deflection of the substrate, $\Delta z$, can be approximated by a double-clamped beam ${ }^{26}$

$$
r=\Delta d / \Delta z=6 \cdot t \cdot s / D^{2} .
$$

with $s$ being the total thickness of the substrate (metal substrate and polymer stack), $t$ the distance between the antenna's anchoring points on the substrate, and $D$ the distance between the fixed supports of the bending mechanism (Fig. 1A). Most systems attain values for $r$ between $10^{-4}$ and $10^{-5}$, exemplifying the high mechanical reduction factor of MCBJs. In practice, inhomogeneities in the elastic properties of the polymer-metal stack ${ }^{32}$ on top of the substrate affect equation (1). In MCBJs, electrical signals can be used for estimating the distance between the electrodes, ${ }^{33}$ as the resistance of the junction, $R$, follows an exponential dependence on $\Delta d$ with the relationship described by Simmon's tunneling law. ${ }^{34}$ To leverage the MCBJ approach for our optical purposes, however, electrical leads need to be attached to antennas to enable electrical control of the gap sizes. Accordingly, we term the resulting system "mechanically actuated plasmonic antenna" (MAPA). The lead placement aims at maintaining optical resonance conditions under incident, monochromatic laser light and strong near-field enhancement. Numerical simulations were performed to vary the design parameters to obtain the optimal configuration (see SI). By placing the leads at the nodes of the plasmonic mode distribution (Fig. 1B), the highest field-enhancements and lowest SPP disturbances are attained. The overall length of the antenna, $L$, is set to $80 \mathrm{~nm}$ and $160 \mathrm{~nm}$ (to achieve resonant conditions for $632 \mathrm{~nm}$ excitation) and the lead width, $u$, to $40 \mathrm{~nm}$ (Fig. S2C). Eight antennas with corresponding leads are placed on a single chip (Fig. 1D). To experimentally validate the simulations, we fabricated closed (with a $10 \mathrm{~nm}$ wide constriction) and open (10 nm gap) antennas with and without leads on transmission-electron microscopy (TEM)compatible, rigid substrates (see SI) as schematically illustrated in Fig. 2A. Feature sizes (for both gaps and constrictions) below that range turned out to be less reproducible, mainly because of grains and voids present in the evaporated Au. Electron energy-loss spectroscopy (EELS) was performed using an aberration-corrected FEI Titan equipped with a gun monochromator (see SI) to map the entire distribution of plasmon modes across the optical structure. ${ }^{13,35,36}$ The antennas were excited with the electron beam focused on different locations of the antenna, as marked in Fig. $2 \mathrm{~A}$, to induce distinct charge distributions. Fig. $2 \mathrm{~B}$ and $2 \mathrm{C}$ show EELS spectra and maps of antennas in the absence and presence of electrical leads, respectively. A scanning TEM (STEM) image of the respective antennas is shown as an inset in each plot. From the EELS spectra of both type of antennas, four distinct resonant peaks can be identified, corresponding to the dipolar bright mode (BM), the quadrupolar mode (QM), and the dipolar dark mode (DM), and a peak that is formed by the coupling of QMs and edge modes. ${ }^{37-39}$ Owing to the proximity of these modes, separated in some cases only by $40 \mathrm{meV}$, and the relatively low effective energy resolution of approx. $90 \mathrm{meV}$, as well as the weak coupling of the QM and edge modes of each triangular sub-antenna, we observe only one peak for these coupled modes (CMs). EELS simulations using the boundary element method (MNPBEM toolbox) ${ }^{40,41}$ were performed (see SI). Comparing the EELS spectra of the antenna with and without electrical leads, as summarized in Fig. 2D (the dotted lines show the experimentally derived energy shifts for open antennas with and without leads), we observe that neither of the modes disappears completely and that the shifts were smaller than $90 \mathrm{meV}$ upon lead attachment for all modes. Furthermore, the EELS maps of the antenna with and without leads (Fig. 2B and 2C) show no qualitative difference for BM, DM, or QM excitations. Only small differences in the maps of the CMs (see SI) are observed as CMs have anti-nodes at the lead attachment positions. Hence, the symmetric positioning of the leads chosen is optimal and guarantees the conservation of the symmetry of the modal structure. To summarize the simulations and EELS experiments, the design chosen for the lead attachment barely alters plasmonic modes, and only a small blue shift of less than $100 \mathrm{meV}$ is observed. Additionally, the intensity of the dipolar bright mode seems to be similarly high with and without leads, which allows high field enhancements to be expected also for "wired" antennas.

For electro-mechanical measurements, freestanding MAPAs are fabricated on flexible Beryllium-Copper $(\mathrm{BeCu})$ substrates as the elastic bending range of the electron-transparent $\mathrm{Si}_{x} \mathrm{~N}_{y}$ membranes is not sufficient to break the constriction of the antennas. Furthermore, a flexible nanoimprint polymer (NIP) of approx. $9 \mu \mathrm{m}$ thickness is used as an electrically insulating layer, creating a different dielectric environment below the antennas (with a refractive index $n=1.52 @ 589 \mathrm{~nm})$ compared to $\mathrm{Si}_{x} \mathrm{~N}_{y}(n=2.05 @ 600$ $\mathrm{nm}$ ) used for the EELS studies. The antennas become elevated in respect to the NIP by a plasma etch, which concentrates the strain on the freestanding segment and further enhances optical properties. ${ }^{42}$ These devices are then mounted in a three-point bending mechanism with $D=11.4 \mathrm{~mm}$, as shown in Fig. 1A. A Newport SR50CC rotatory motor actuates the substrate and electrical signals are acquired with a Hewlett Packard 4155C Semiconductor Parameter Analyzer at a probing voltage of $5 \mathrm{mV}$. As displayed by the electrical resistance of the junction between the two antenna segments, $R$, the gap can repeatedly be opened and closed by bending the substrate in tensile direction and bending it back again (Fig. $3 \mathrm{~A})$; distinct changes between a closed $(\mathrm{R}<1 \mathrm{k} \Omega)$ and an open $(\mathrm{R}>10 \mathrm{G} \Omega$ ) junction occur at reproducible motor positions (in motor position degrees, ${ }^{\circ}$; with little drift $\left(<120^{\circ} / 500\right.$ cycles $)$ and a consistent mechanical hysteresis (e.g. $(100 \pm 20)^{\circ}$ for $1 \mathrm{k} \Omega$ and $10 \mathrm{G} \Omega$ as electrically controlled reversal points; Fig. 3B). Apart from this binary opening-closing operation, arbitrary resistances between the fully closed and the fully opened configuration can be set (upper panel in Fig. 3C) by adjusting the position accordingly (lower panel in Fig. 3C). The junction's long-term stability at room temperature is low for the breaking case, due to spontaneous drifts occurring by the strain release in the NIP. In contrast, the stability increases for the closing case, as strain is now absent and the material relaxed. The best stability is found for either completely open or fully closed junctions, as evidenced for the quantum-point-contact regime around $1 \cdot \mathrm{G}_{0}$ acquired at $300 \mathrm{~K}$ (Fig. 3D) with $\mathrm{G}_{0}$ being the conductance quantum. Please consult the various chapters of the SI regarding the gap-size estimation displayed in Fig. 3F which is based on electron tunneling traces (Fig. 3E), mechanical considerations and direct strain-gauge measurements. The gap modulation of the MAPAs enables various plasmonic coupling regimes and field-enhancements to be created and fine-tuned, as illustrated in Figs. $1 \mathrm{C}$ and $3 \mathrm{C}$.

With the mechanical gap tunability of the MAPAs at hand, optical characterization is then performed using a custom-made dark-field microscope with a spot-size below $1 \mu \mathrm{m}$ (see SI) to probe single antennas. As absorption-induced heating effects prevented so far conventional MCBJs from being used as gap-tunable optical structures, we first study broad-band optical excitation of our tiny MAPAs and its influence on electron tunneling across the gaps. Fig. 4A shows time evolutions of the junction's resistance upon illuminating individual antennas with broad-band, pulsed light (SuperK Compact super-continuum laser from NKT Photonics 
with an emission from $450 \mathrm{~nm}$ to $2400 \mathrm{~nm}$, and an Olympus type MPLN10x objective). The junction's conductance is initially set to values of approx. $10^{-4} \cdot \mathrm{G}_{0}$ to cover the optically relevant quantum-tunneling regime. ${ }^{9}$ The incident laser power is measured directly at the sample plane using a $\mathrm{Si}$ photodiode-based power meter inside a light-integrating sphere (Thorlabs Type PM16-140) at $600 \mathrm{~nm}$, and may be substantially higher given the broad-band incident light and broad-band absorption of Au. The laser intensity was progressively increased from 0 to $3.2 \mathrm{~kW} / \mathrm{cm}^{2}$, with the highest intensity being approx. 10x stronger than that required to record a scattering spectrum $\left(0.32 \mathrm{kw} / \mathrm{cm}^{2}\right)$ with reasonable signal-to-noise ratio as discussed later. While the junction's resistance remains almost constant over 200 seconds of illumination, the relative peak-to-peak fluctuation variation increases slightly with increasing laser power (Fig. 4B). Apart from these increased tunneling current fluctuations, which are most likely caused by an increased surface mobility of Au atoms rather than the net thermal expansion of the antenna halves, the MAPAs seem to provide the required long-term stability under relevant illumination intensities.

When now performing opening and closing cycles of the gaps as shown in Fig. 3A, we observe a transversal drift of $(200-1200)$ $\mathrm{nm} / 500^{\circ}$ of the antenna's position in respect to the optical axis that is accompanied by focal changes of $(200-500) \mathrm{nm} / 500^{\circ}$, caused by the Poisson effect inside the NIP. As a consequence, optical data was gathered step-by-step rather than continuously, after intermediate refocusing. Fig. 4C displays selected, single-antenna scattering spectra (see SI) acquired with a thermo-electrically cooled Ocean Optic QE Pro High Performance back-thinned Si-CCD Spectrometer (groove density $300 \mathrm{~mm}^{-1}, 400-1100 \mathrm{~nm}$ spectral range; $1.98 \mathrm{~nm}$ spectral resolution) at $0.32 \mathrm{kw} / \mathrm{cm}^{2}$ and smoothed by a FFTF process to remove the short-wavelength cavity resonances (see SI). At least 10 opening and closing cycles are performed for each device and multiple spectra taken per position. Spectral data is selected according to the electrical conductance traces acquired simultaneously (see SI). Starting with open gaps larger than (1.0 \pm $0.25) \mathrm{nm}$ (dark blue spectra in Fig. 4C), the background-corrected spectrum reveal a broad resonance centered at $(627 \pm 15) \mathrm{nm}$ (average over 20 cycles) with a FWHM of approx. $65 \mathrm{~nm}$. Superimposed on the main peak are spectrally periodic modulations, which are residuals from the FFTF process. The antennas main resonance matches the targeted value for monochromatic excitation at $632 \mathrm{~nm}$, chosen both for the strain-induced experiments with expected red-shifts (see below) and future Raman-scattering experiments. While closing the junction, the main resonance (indicated as non-matching Gaussians as a guide-to-the-eye only) gradually red-shifts to values around $(677 \pm 10) \mathrm{nm}$ at gap sizes of $(0.4 \pm$ $0.1) \mathrm{nm}$ (spectra shifted by 4 a.u.). Upon further reducing the gap size beyond that point, the spectra's maxima move back towards smaller wavelengths and spectral changes occur more abruptly and distinctly. While in the near-to-contact regime, the junction is less stable, as discussed above and indicated by larger fluctuations in the corresponding tunneling trace displayed in Fig. 4D (with the colored dots indicating the corresponding spectra in Fig. 4C), both electrical and optical signals become stable again when full metallic contact is established (brown data). Here, the resonance is again pronounced with a reasonable signal-to-noise ratio of 5, located, however, at lower wavelengths of $(580 \pm 20) \mathrm{nm}$ than initially. Potentially a second mode evolved at around $(760 \pm 8) \mathrm{nm}$. Due to the highly dynamic behavior at sub- $0.1 \mathrm{~nm}$ separations, e.g. the spontaneous jump-to-contact behavior, we refrain from displaying corresponding spectra for this near-to-contact range. Tracking the broad main resonance feature (highlighted by the color-changing lines in Fig. 4C, based on Gaussian fits) shows a more continuous shift than considering the spectral maxima (black dotted lines), which are assumed to be subject to a cavity-mediated amplification process. By repeating these experiments on 20 samples, we find a good overall reproducibility of the shifts as summarized in Fig. 4E (the motor positions are reproducible within $\pm 10^{\circ}$ ). Even though our unpolarized dark-field experiment can currently only track the main scattering resonance of the antennas (and spatially averages over all polarization-dependent higher-order or hybrid modes), the resonance shift upon closing the gap is similar to previous findings in variable-gap plasmonic antennas. ${ }^{9}$ The onset of abrupt spectral shifts occurs at around $0.25 \mathrm{~nm}-0.35 \mathrm{~nm}$, and distinctly different spectra appear after having created a plasmonic "short". The exchange of charge carriers should make the dipolar mode disappear. A charge-transfer or threaded dipolar plasmon ${ }^{43}$ is supposed to appear instead. We assume the new mode appearing at $(580 \pm 20) \mathrm{nm}$ to be this charge-transfer plasmon (and the mode at $(760 \pm 8) \mathrm{nm}$ its higher-order representative, consequently weaker in intensity). Unfortunately, microscopic details of "shorted" antennas cannot be inspected by SEM or TEM to determine the junction that formed, which would allow the strength of the charge-transfer effect to be estimated. By implementing a mechanical drift-correcting algorithm, based on piezoelectric actuation, we hope to provide comprehensive data sets of continuously varied plasmonic coupling experiments in the near future where also subtle features can be monitored. To determine the different charge-carrier distributions and to disentangle various plasmonic modes, polarizers are currently being implemented. Even though such further improvements are still required, we believe that the MAPA approach presented may enable future in-depth electro-optical investigations of plasmonic field-enhanced devices as a function of inter-electrode spacing, e.g. for field-enhanced Raman experiments.

In conclusion, we have presented a novel approach that combines resonant optical antennas with mechanical strain actuation and electrical control to mechanically vary the gaps of optical antennas. The device layout enables antennas to be electrically contacted by leads while only weakly perturbing the plasmonic near-fields but enabling electrical control of gap sizes in return. MAPAs can reproducibly be opened and closed under ambient conditions, which facilitates experiments and broadens the application range. Optical scattering spectra reveal the expected spectral shifts and discrete changes at sub-1nm gaps and shorted antennas in simultaneous electro-optical experiments. MAPAs are considered a promising platform for investigations of optical processes in high-field enhancement structures that can further be electrically biased for various purposes.

\section{ASSOCIATED CONTENT}

Supporting Information on the fabrication, simulation, EELS, the electro-mechanical setup, low-temperature and UHV data, local strain gauges, spectral background and FFTF-based data processing as well as tunneling data and statistics. This material is available free of charge via the Internet at http://pubs.acs.org.

\section{AUTHOR INFORMATION}

Corresponding Author: eml@ zurich.ibm.com (E.L.)

\section{NOTES}

The authors declare no competing financial interest.

\section{ACKNOWLEDGMENTS}


We are grateful to M. Raschke, O. Martin, G.-L. Bona, and E. Scheer for scientific discussions, and M. Tschudy, M. Bürge, A. Zulji, U. Kloter and P. Wälchli for technical assistance. We thank B. Michel, W. Riess, H. Riel and A. Curioni for continuous support. Funding from the Swiss National Science Foundation (Grant CR22I2-152944) is gratefully acknowledged. E.B.P. and G.A.B. acknowledge support from NSERC under the Discovery Grant Program. The EELS work was carried out at the Canadian Centre for Electron Microscopy, a National Facility supported by The Canada Foundation for Innovation under the MSI program, NSERC, and McMaster University.

\section{References}

(1) Nie, S.; Emory, S. R. Probing single molecules and single nanoparticles by surface-enhanced Raman scattering. Science 1997, 275, 1102.

(2) Xu, H.; Bjerneld, E. J.; Käll, M.; Börjesson, L. Spectroscopy of single hemoglobin molecules by surface enhanced Raman scattering. Physical Review Letters 1999, 83, 4357.

(3) Michaels, A. M.; Nirmal, M.; Brus, L. E. Surface Enhanced Raman Spectroscopy of Individual Rhodamine 6G Molecules on Large Ag Nanocrystals. Journal of the American Chemical Society 1999, 121, 9932.

(4) Taylor, R. W.; Lee, T.-C.; Scherman, O. A.; Esteban, R.; Aizpurua, J.; Huang, F. M.; Baumberg, J. J.; Mahajan, S. Precise Subnanometer Plasmonic Junctions for SERS within Gold Nanoparticle Assemblies Using Cucurbit[n]uril Glue. ACS Nano 2011, 5, 3878-3887.

(5) Ciracì, C.; Hill, R. T.; Mock, J. J.; Urzhumov, Y.; FernándezDomínguez, A. I.; Maier, S. A.; Pendry, J. B.; Chilkoti, A.; Smith, D. R. Probing the Ultimate Limits of Plasmonic Enhancement. Science 2012, 337, 1072.

(6) Aimovi, S. S.; Ortega, M. A.; Sanz, V.; Berthelot, J.; Garcia-Cordero, J. L.; Renger, J.; Maerkl, S. J.; Kreuzer, M. P.; Quidant, R. LSPR Chip for Parallel, Rapid, and Sensitive Detection of Cancer Markers in Serum. Nano Lett. 2014, 14, 2636-2641.

(7) Yavas, O.; Aimovi, S. S.; Garcia-Guirado, J.; Berthelot, J.; Dobosz, P.; Sanz, V.; Quidant, R. Self-Calibrating On-Chip Localized Surface Plasmon Resonance Sensing for Quantitative and Multiplexed Detection of Cancer Markers in Human Serum. ACS Sens. 2018, 3, 1376-1384.

(8) Zhu, W.; Crozier, K. B. Quantum mechanical limit to plasmonic enhancement as observed by surface-enhanced Raman scattering. Nature Coтmunications 2014, 5, 1.

(9) Savage, K. J.; Hawkeye, M. M.; Esteban, R.; Borisov, A. G.; Aizpurua, J.; Baumberg, J. J. Revealing the quantum regime in tunnelling plasmonics. Nature 2012, 491, 574.

(10) Schmidt, M. K.; Esteban, R.; González-Tudela, A.; Giedke, G.; Aizpurua, J. Quantum Mechanical Description of Raman Scattering from Molecules in Plasmonic Cavities. ACS Nano 2016, 10, 6291.

(11) Roelli, P.; Galland, C.; Piro, N.; Kippenberg, T. J. Molecular cavity optomechanics as a theory of plasmon-enhanced Raman scattering. Nature Nanotechnology 2015, 11, 164.

(12) Chikkaraddy, R.; de Nijs, B.; Benz, F.; Barrow, S. J.; Scherman, O. A.; Rosta, E.; Demetriadou, A.; Fox, P.; Hess, O.; Baumberg, J. J. Singlemolecule strong coupling at room temperature in plasmonic nanocavities. Nature 2016, 535, 127.

(13) Koh, A. L.; Fernandez-Dominguez, A. I.; McComb, D. W.; Meier, S. A.; Yang, J. K. W. High-Resolution Mapping of Electron-Beam-Excited Plasmon Modes in Lithographically Defined Gold Nanostructures. Nano Letters 2010, 11, 1323.

(14) Zhao, J.; Frank, B.; Burger, S.; Giessen, H. Large-Area High-Quality Plasmonic Oligomers Fabricated by Angle-Controlled Colloidal Nanolithography. ACS Nano 2011, 5, 9009.

(15) Lee, S.-W.; Lee, K.-S.; Ahn, J.; Lee, J.-J.; Kim, M.-G.; Shin, Y.-B. Highly
Sensitive Biosensing Using Arrays of Plasmonic Au Nanodisks Realized by Nanoimprint Lithography. ACS Nano 2011, 5, 897.

(16) Mühlschlegel, P.; Eisler, H.-J.; Martin, O. J. F.; Hecht, B.; Pohl, D. W. Resonant Optical Antennas. Science 2005, 308, 1607.

(17) Herzog, J. B.; Knight, M. W.; Li, Y.; Evans, K. M.; Halas, N. J.; Natelson, D. Dark Plasmons in Hot Spot Generation and Polarization in Interelectrode Nanoscale Junctions. Nano Letters 2013, 13, 1359.

(18) Ittah, N.; Yutsis, I.; Selzer, Y. Fabrication of Highly Stable Configurable Metal Quantum Point Contacts. Nano Lett. 2008, 8, 3922-3927.

(19) Bragas, A. V.; Landi, S. M.; Martínez, O. E. Laser field enhancement at the scanning tunneling microscope junction measured by optical rectification. Applied Physics Letters 1998, 72, 2075.

(20) Schneider, N. L.; Schull, G.; Berndt, R. Optical Probe of Quantum ShotNoise Reduction at a Single-Atom Contact. Physical Review Letters 2010, 105, 026601 .

(21) Schull, G.; Néel, N.; Johansson, P.; Berndt, R. Electron-Plasmon and Electron-Electron Interactions at a Single Atom Contact. Physical Review Letters 2009, 102, 057401.

(22) Merlein, J.; Kahl, M.; Zuschlag, A.; Sell, A.; Halm, A.; Boneberg, J.; Leiderer, P.; Leitenstorfer, A.; Bratschitsch, R. Nanomechanical control of an optical antenna. Nature Photonics 2008, 2, 230.

(23) Scholl, J. A.; García-Etxarri, A.; Koh, A. L.; Dionne, J. A. Observation of quantum tunneling between two plasmonic nanoparticles. Nano Letters 2013, 13, 564.

(24) Yanson, A. I.; Rubio Bollinger, G.; Van den Brom, H. E.; Agraït, N.; van Ruitenbeek, J. M. Formation and manipulation of a metallic wire of single gold atoms. Nature 1998, 395, 783.

(25) Agraï, N.; Yeyati, A. L.; van Ruitenbeek, J. M. Quantum properties of atomic-sized conductors. Physics Reports 2003, 377, 81.

(26) van Ruitenbeek, J. M.; Alvarez, A.; Piñeyro, I.; Grahmann, C.; Joyez, P.; Devoret, M. H.; Esteve, D.; Urbina, C. Adjustable nanofabricated atomic size contacts. Review of Scientific Instruments 1996, 67, 108.

(27) Schwarz, F.; Lörtscher, E. Break-junctions for investigating transport at the molecular scale. J. Phys.: Condens. Matter 2014, 26, 474201

(28) Tian, J.-H.; Liu, B.; Li, X.; Yang, Z.-L.; Ren, B.; Wu, S.-T.; Tao, N.; Tian, Z.-Q. Study of molecular junctions with a combined surfaceenhanced Raman and mechanically controllable break junction method. Journal of the American Chemical Society 2006, 128, 14748.

(29) Konishi, T.; Kiguchi, M.; Takase, M.; Nagasawa, F.; Nabika, H.; Ikeda, K.; Uosaki, K.; Ueno, K.; Misawa, H.; Murakoshi, K. Single Molecule Dynamics at a Mechanically Controllable Break Junction in Solution at Room Temperature. Journal of the American Chemical Society 2012, 135, 1009.

(30) Alexander, K. D.; Skinner, K.; Zhang, S.; Wei, H.; Lopez, R. Tunable SERS in Gold Nanorod Dimers through Strain Control on an Elastomeric Substrate. Nano Letters 2010, 10, 4488.

(31) Novotny, L. Effective Wavelength Scaling for Optical Antennas. Phys. Rev. Lett. 2007, 98, 266802.

(32) Vrouwe, S. A. G.; van der Giessen, E.; van der Molen, S. J.; Dulic, D.; Trouwborst, M. L.; van Wees, B. J. Mechanics of lithographically defined break junctions. Physical Review B 2005, 71, 035313.

(33) Martin, C. A.; Ding, D.; van der Zant, H. S. J.; van Ruitenbeek, J. M. Lithographic mechanical break junctions for single-molecule measurements in vacuum: possibilities and limitations. New Journal of Physics 2008, 10, 065008.

(34) Simmons, J. G. Generalized formula for the electric tunnel effect between similar electrodes separated by a thin insulating film. Journal of Applied Physics 1963, 34, 1793.

(35) Duan, H.; Fernández-Domínguez, A. I.; Bosman, M.; Maier, S. A.; Yang, J. K. Nanoplasmonics: classical down to the nanometer scale. Nano Letters 2012, 12, 1683.

(36) Bosman, M.; Keast, V. J.; Watanabe, M.; Maaroof, A. I.; Cortie, M. B. Mapping surface plasmons at the nanometre scale with an electron beam. Nanotechnology 2007, 18, 165505. 
(37) Schmidt, F.-P.; Ditlbacher, H.; Hohenester, U.; Hohenau, A.; Hofer, F.; Krenn, J. R. Universal dispersion of surface plasmons in flat nanostructures. Nature Communications 2014, 5, 3604.

(38) Bellido, E. P.; Manjavacas, A.; Zhang, Y.; Cao, Y.; Nordlander, P.; Botton, G. A. Electron Energy-Loss Spectroscopy of Multipolar Edge and Cavity Modes in Silver Nanosquares. ACS Photonics 2016, 3, 428.

(39) Bellido, E. P.; Zhang, Y.; Manjavacas, A.; Nordlander, P.; Botton, G. A. Plasmonic Coupling of Multipolar Edge Modes and the Formation of Gap Modes. ACS Photonics 2017, 4, 1558.

(40) Hohenester, U.; Trügler, A. MNPBEM - A Matlab toolbox for the simulation of plasmonic nanoparticles. Computer Physics Communications 2012, 183,370

(41) Hohenester, U. Simulating electron energy loss spectroscopy with the MNPBEM toolbox. Computer Physics Communications 2014, 185, 1177.

(42) Hatab, N. A.; Hsueh, C.-H.; Gaddis, A. L.; Retterer, S. T.; Li, J.-H.; Eres, G.; Zhang, Z.; Gu, B. Free-Standing Optical Gold Bowtie Nanoantenna with Variable Gap Size for Enhanced Raman Spectroscopy. Nano Lett. 2010, 10, 4952.

(43) Herrmann, L. O.; Valev, V. K.; Tserkezis, C.; Barnard, J. S.; Kasera, S.; Schermann, O. A.; Aizpurua, J.; Baumberg, J. J. Threading plasmonic nanoparticle strings with light. Nat. Communications 2014, 5, 4568. 
A Mechanical Actuation and Junction-forming Principle

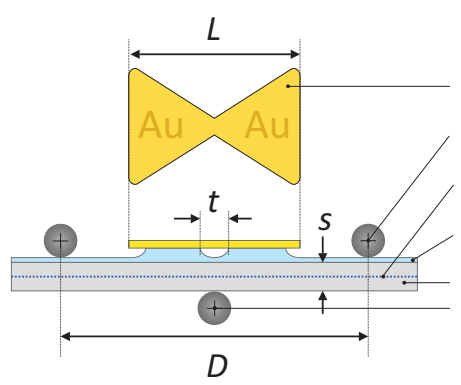

Neutral $(\varepsilon=0)$

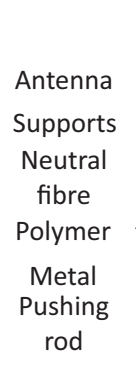

rod

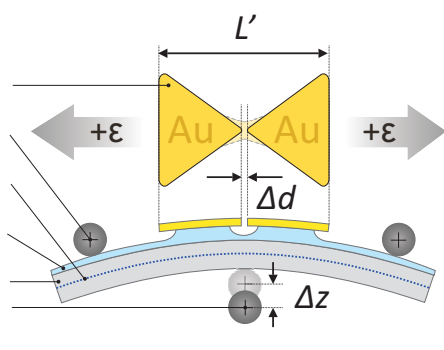

Tensile Strain $(\varepsilon>0)$
B Antenna Design w/ Leads

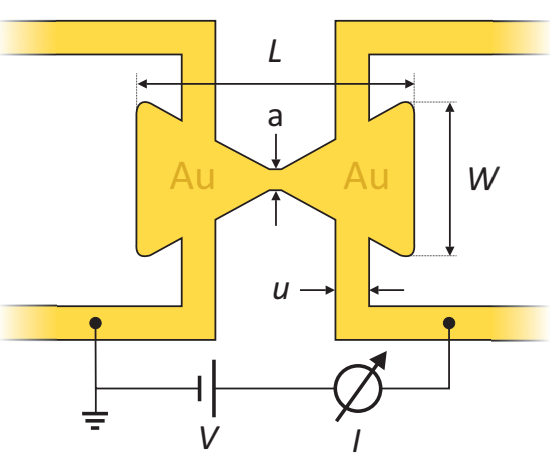

D Chip Mounted in Setup

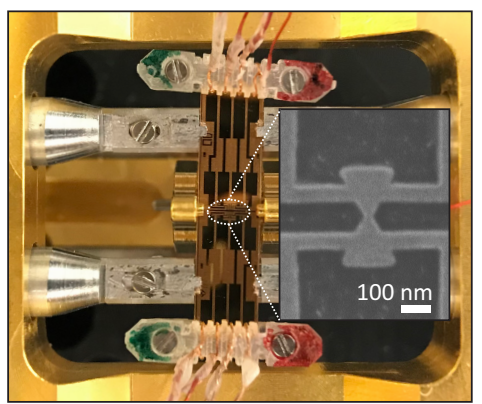

Figure 1. A) Mechanical actuation principle to break a constriction within the gap region of the antenna. Tensile strain ( $\varepsilon>0$ ) is induced by bending the substrate via a pushing rod. By lowering the pushing rod and reducing the tensile strain the junction can be closed again. As dimensions are not to scale and the distance of the plasmonic antenna to the neutral fiber is much smaller than the bending radius of the substrate, the strain applied to the antenna is considered to be uniaxial on the scale of the antenna. B) Schematics of the different microscopic junction configurations achieved by mechanical stretching of the initial bulky contact (left). The ductile response of Au upon deformation yields atomic-sized leads with quantum point contacts (QPC) just before breaking (middle). Upon further bending, the junction can finally be broken to establish a tunneling junction and to ultimately separate the plasmonic sub-systems. This process is fully reversible and can be repeated several hundred to thousand times. C) Overview of the antenna's design parameters including electrical leads attached in a way to minimize plasmon resonance disturbances (see SI). D) Top view of the bending mechanism with a chip mounted and being contacted by electrical clamps (red wires). Each device contains eight antennas oriented as depicted by the SEM inset. 
A Antenna Stack / EELS Excitation Points
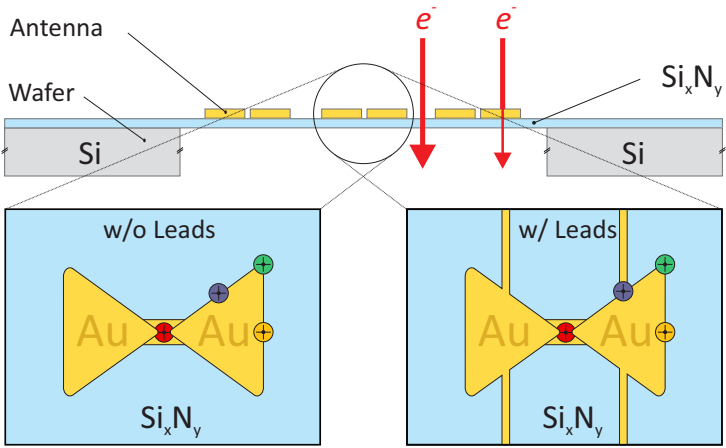

B EELS Spectra and Plasmon Maps (w/o Leads)

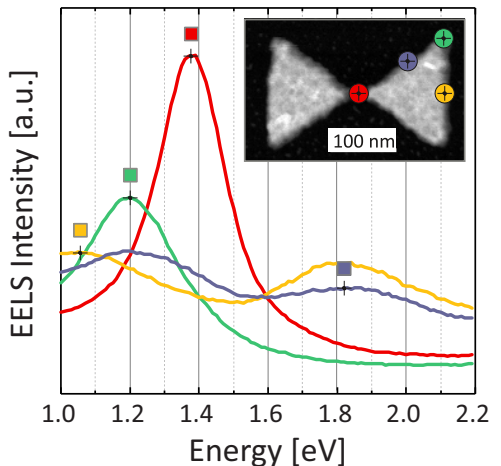

D Mode Energy Shift upon Lead Attachment

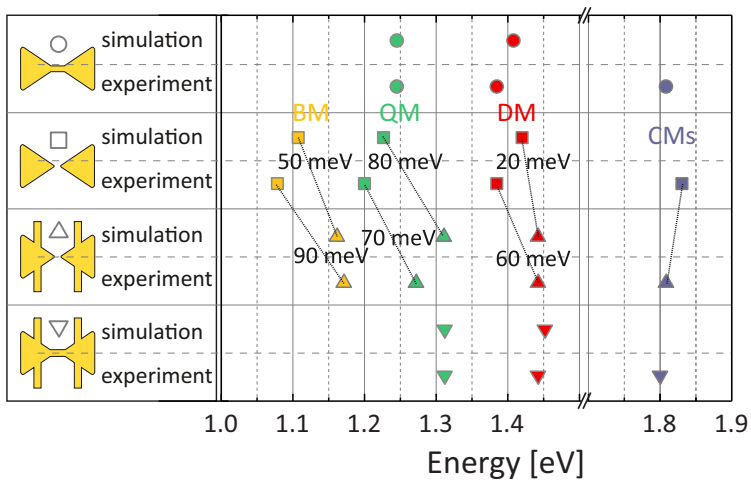

EELS Spectra and Plasmon Maps (w/ Leads)

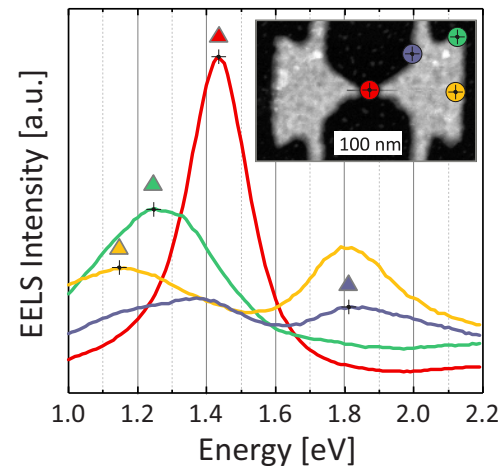

Figure 2. A) Antennas are fabricated on electron-transparent, $30 \mathrm{~nm}$ thick and suspended $\mathrm{Si}_{x} \mathrm{~N}_{y}$ membranes for EELS experiments by TEM. During investigations, antennas without and with leads are probed at different locations to excite distinct plasmon modes: Gap or constriction-centered excitation (red) induces charge-carrier excitations that represent dipolar dark modes (DM), excitation at the outer corners (green) dipolar bright modes (BM), and excitations at the diagonal edge (purple) and the edge opposite the gap (yellow) quadrupolar DM. EELS spectra and energy-dependent plasmon maps of single open antennas without (B) and with electrical lead attachments (C). D) Summary of the experimentally derived energy shifts of all relevant plasmonic modes of open and closed antennas without and with leads (compared to simulations; upper panels). It reveals only small energy shifts below $100 \mathrm{mV}$ for the relevant optical modes. 
A Repeated Junction Opening and Closing Cycles (300 K)

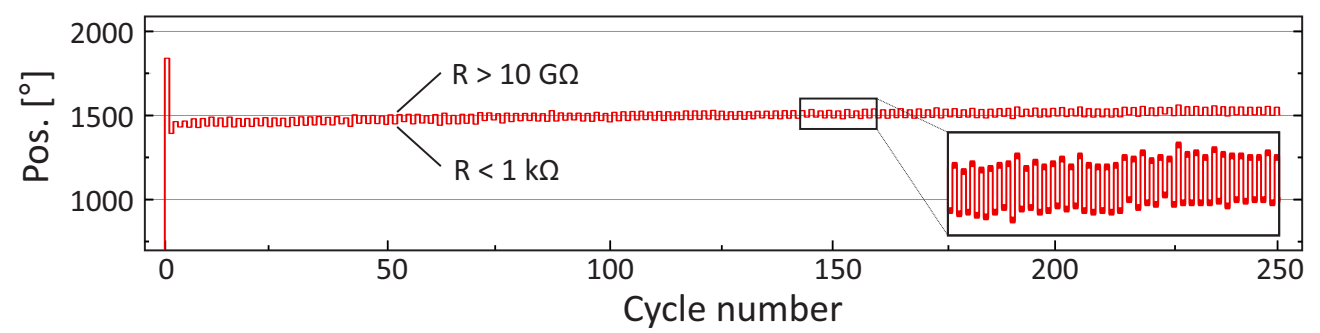

B Hysteresis

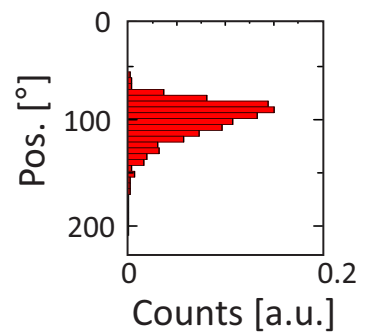

C Dynamic Range and Static Stability
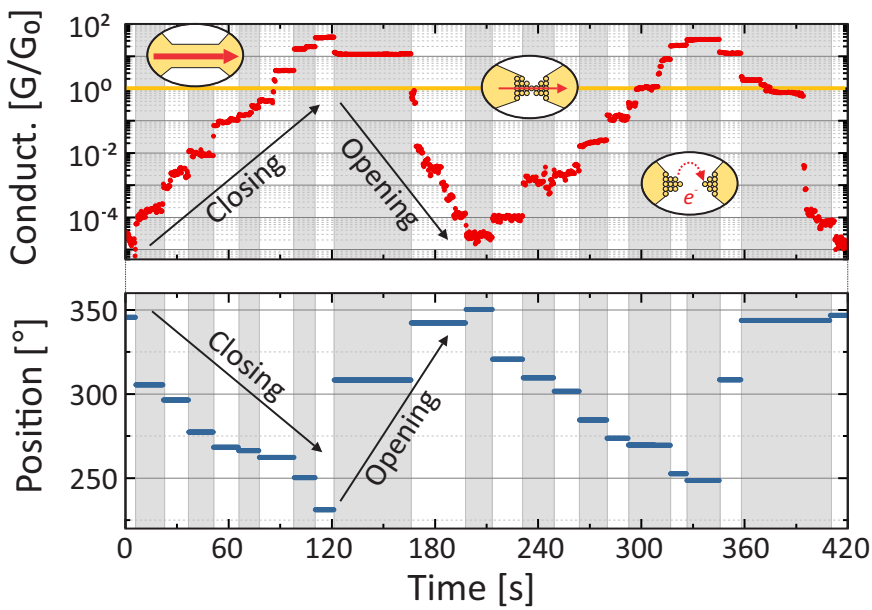

E Electron Tunnelling Closing Traces

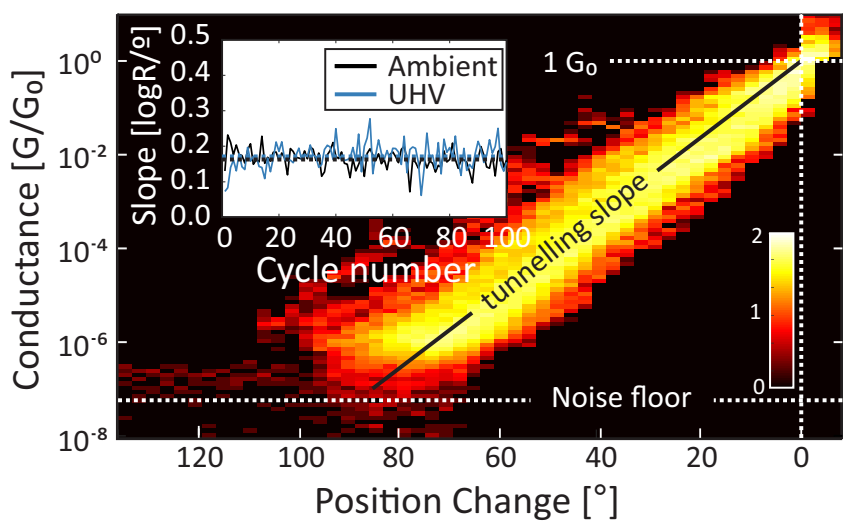

D Stability in the QPC Regime

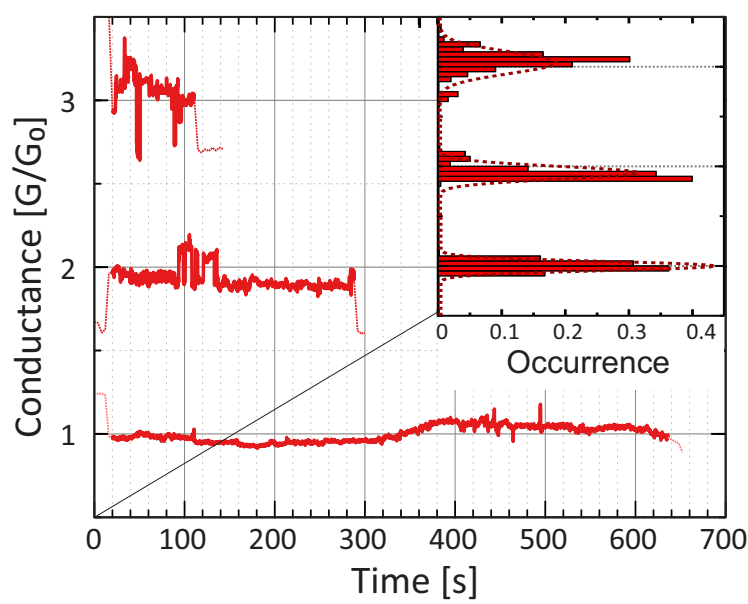

F Gap-size Estimation

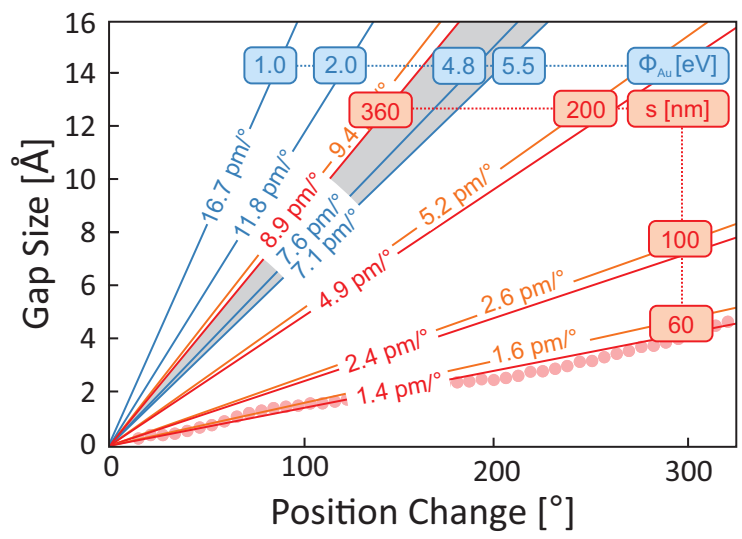

Figure 3. A) Repeated opening and closing of the junction with (B) reproducible hysteresis between opening and closing positions. C) Adjustment of arbitrary junction resistances (upper panel) by adjusting the motor accordingly (lower panel). A long-term stability is only given for closing traces at $300 \mathrm{~K}$. For opening the junction, strain applied to the NIP relaxes on longer times scales. D) Enhanced stability in the quantum-point-contact regime. E) Electron-tunneling closing traces with a similar behavior as operation under UHV conditions (inset). F) Slopes for the gap size as a function of control position change, calculated from Simmon's tunneling law assuming various work functions (blue) and calculated from local strain gauges assuming various undercut dimensions (red slopes indicate resistive, orange slopes capacitive data, respectively; light red dots show the underlying resistive-gauge measurement data). 
A Junction Resistance under varying Illumination Intensities (300 K)

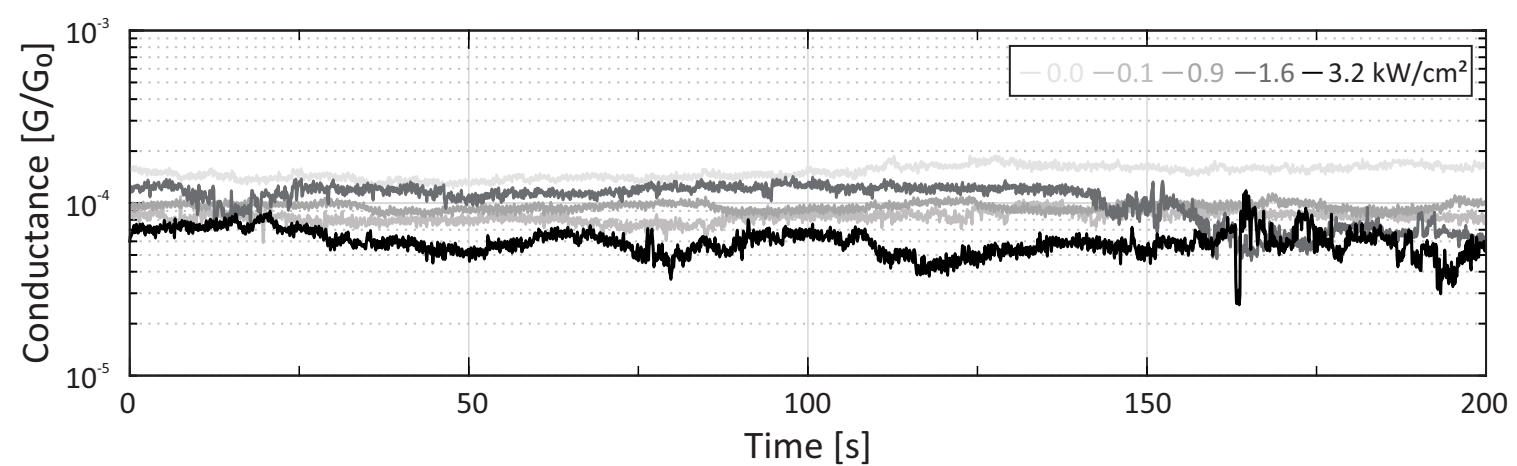

B Resistance Variation

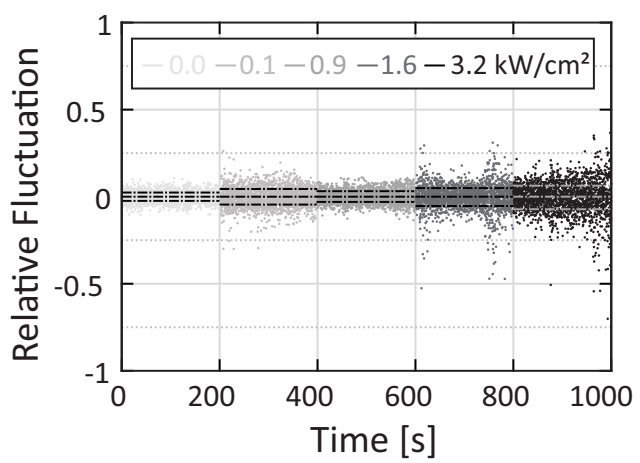

D Conductance Trace

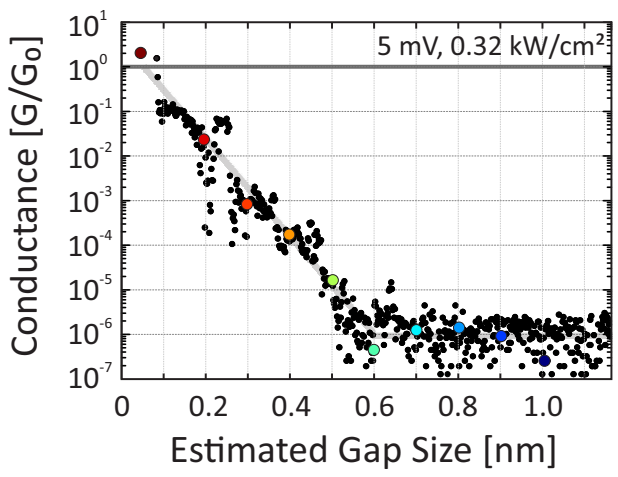

E Main Resonance Shift

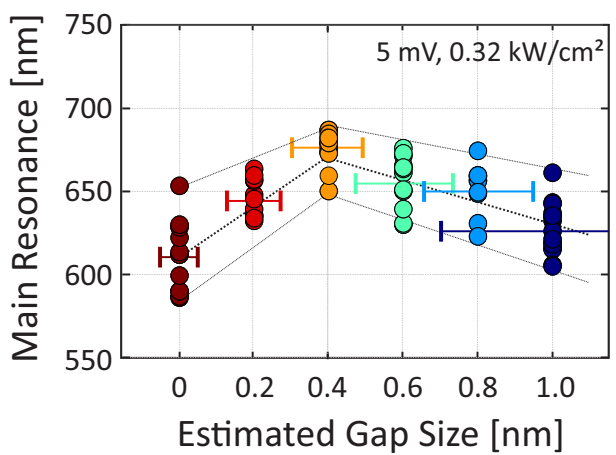

C Gap-dependent Scattering Spectra

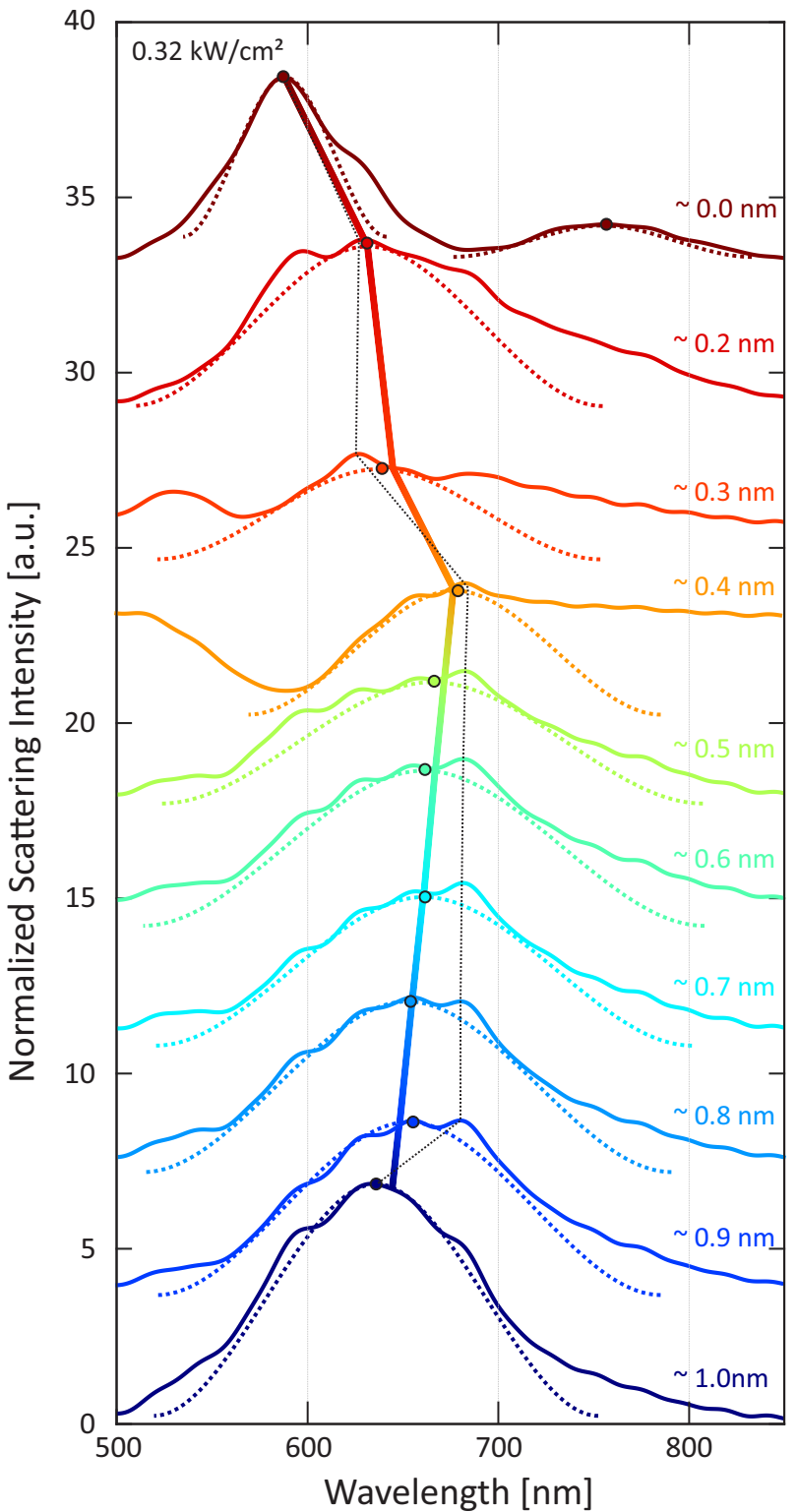

Figure 4. A) Tunneling currents under varying, broad-band illumination power from 0 to $3.2 \mathrm{~kW} / \mathrm{cm}^{2}$ (measured for $600 \mathrm{~nm}$ with a bandpass of $25 \mathrm{~nm}$ ). B) Relative conductance fluctuations. C) Scattering spectra after FFTF smoothing (see SI), measured for an individual antenna with an $80 \mathrm{~nm}$ triangle size and varying gap sizes upon strain modulation. The main resonance is moving towards longer wavelengths up to a gap size of about $0.4 \mathrm{~nm}$ when starting from a fully open antenna. At that point, the resonance is blue-shifting again with an abrupt spectral change appearing when getting in electrical contact. D) Simultaneously recorded electrical conductance as a function of estimated gap size with the colored dots indicating the scattering spectra shown in C). E) Statistics of the main resonance shift extracted from 20 samples. 


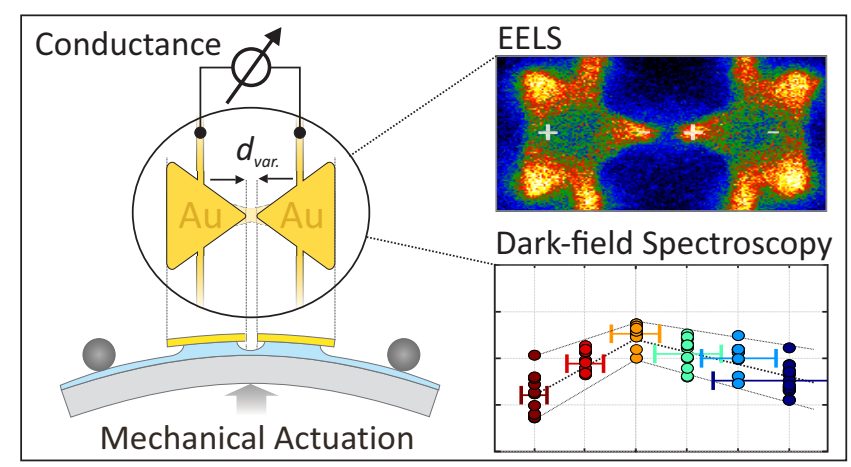

Figure 5. Table of contencts graphic: For Table of Contents Only. 